\title{
Hand-Foot Syndrome and Post-Progression Treatment Are the Good Predictors of Better Survival in Advanced Hepatocellular Carcinoma Treated with Sorafenib: A Multicenter Study
}

\author{
Chikara Ogawa ${ }^{a}$ f Masahiro Morita ${ }^{a}$ Akina Omura ${ }^{a}$ Teruyo Noda ${ }^{a}$ \\ Atsushi Kubo $^{a}$ Toshihiro Matsunaka $^{a}$ Hiroyuki Tamaki ${ }^{a}$ \\ Mitsushige Shibatoge $^{a}$ Akemi Tsutsui $^{b}$ Tomonori Senoh $^{b}$ Takuya Nagano $^{b}$ \\ Kouichi Takaguchi $^{\mathrm{b}}$ Joji Tani ${ }^{\mathrm{c}}$ Asahiro Morishitac Hirohito Yoneyamac \\ Tsutomu Masakic Akio Moriyad Masaharu Ando ${ }^{d}$ Akihiro Deguchie \\ Yasutaka Kokudo $^{\text {e }}$ Yasunori Minami $^{f}$ Kazuomi Ueshima ${ }^{f}$ Toshiharu Sakurai $^{f}$ \\ Naoshi Nishida ${ }^{f}$ Masatoshi Kudo $^{f}$

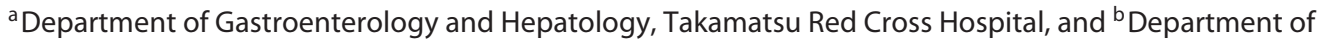 \\ Hepatology, Kagawa Prefectural Central Hospital, Takamatsu, 'Department of Gastroenterology and Neurology, \\ Faculty of Medicine, Kagawa University, Miki, 'Department of Gastroenterology, Mitoyo General Hospital, Kan-onji, \\ e Department of Gastroenterology, Kagawa Rosai Hospital, Marugame, and ${ }^{\mathrm{f}}$ Department of Gastroenterology and \\ Hepatology, Kindai University Faculty of Medicine, Osaka-Sayama, Japan
}

\section{Keywords}

Hepatocellular carcinoma - Sorafenib · Hand-foot syndrome

\begin{abstract}
Objective: To determine the relationship between treatment outcomes and hand-foot syndrome (HFS), and the relationship between survival rate and post-progression treatment after sorafenib therapy. Methods: The study assessed 314 patients with advanced hepatocellular carcinoma (HCC) treated with sorafenib at 5 general hospitals in Kagawa Prefecture, Japan. Results: At the start of sorafenib therapy, $23.6 \%$ of the patients had HCC of a Child-Pugh class other than A. The initial sorafenib dose was $800 \mathrm{mg}$ in $9.2 \%$ of the patients and $400 \mathrm{mg}$ in $64.3 \%$. Time to progression was 129 days (95\% Cl:
\end{abstract}

\section{KARGER}

(C) 2017 S. Karger AG, Basel

E-Mail karger@karger.com

www.karger.com/ocl
87.3-170.7) and the median overall survival (OS) was 392 days (95\% Cl: 316.0-468.0). The OS of the patients with ChildPugh class A HCC was significantly better than that of the patients with Child-Pugh class B HCC $(p<0.0001)$. The survival curves for Child-Pugh class A-5 points and class A-6 points were significantly different, with that for class A-5 points being better $(p<0.0001)$. A significant difference was observed between the patients who exhibited HFS and those who did not, with the former exhibiting a better survival rate $(p<0.001)$. In addition, the survival rate of the patients who received post-progression treatment after sorafenib therapy was significantly better than that of the patients who did not ( $p<0.001)$. Conclusion: In sorafenib therapy, patients with HFS and those who received post-progression treatment exhibited good OS.

(c) 2017 S. Karger AG, Basel
Prof. Masatoshi Kudo

Department of Gastroenterology and Hepatology

Kindai University Faculty of Medicine

377-2 Ohno-Higashi, Osaka-Sayama, Osaka 589-8511 (Japan)

E-Mail m-kudo@med.kindai.ac.jp 


\section{Introduction}

Hepatocellular carcinoma (HCC) is the second leading cause of cancer mortality in the world [1]. Sorafenib is an agent that has improved the time to progression and overall survival (OS) in advanced stages of HCC with vascular invasion and/or extrahepatic spread [2]. Sorafenib has been administered in accordance with the consensusbased treatment algorithm for HCC proposed by the Japan Society of Hepatology [3-6]. According to this algorithm, sorafenib is indicated for patients diagnosed with Child-Pugh class A HCC with extrahepatic spread or vascular invasion. Sorafenib is also recommended for patients with Child-Pugh class A HCC where transcatheter arterial chemoembolization (TACE) [7-11] and hepatic arterial infusion chemotherapy $[12,13]$ are not indicated. In the present study, we examined the efficacy of sorafenib treatment and the clinical outcome in 314 patients at 5 general hospitals in Kagawa Prefecture, Japan.

\section{Subjects and Methods}

Patients

Between June 2009 and January 2016, 314 patients with advanced HCC were treated with sorafenib at 5 general hospitals in Kagawa Prefecture, Japan. The diagnosis of HCC was made based on histological or radiological findings using contrast-enhanced computed tomography and/or dynamic magnetic resonance imaging $[14,15]$. The responses of all patients were evaluated using the modified Response Evaluation Criteria in Solid Tumors (mRECIST) [16].

\section{Statistical Analyses}

Univariate analysis was used to identify predictors of survival using the Kaplan-Meier method, and comparisons were performed using the log-rank test. A $p$ value of $<0.05$ was considered statistically significant. All analyses were performed using SPSS version 22.0 for Windows (IBM, Armonk, NY, USA).

\section{Results}

\section{Baseline Characteristics}

The study included 254 men and 60 women. Their mean age was $72.5 \pm 9.46$ years (range: $38-91$ ), their mean height was $160.4 \pm 9.12 \mathrm{~cm}$ (range: 125.7-179.0), and their mean weight was $58.6 \pm 11.9 \mathrm{~kg}$ (range: $27-101.9$ ). There were 43 patients with hepatitis $\mathrm{B}$ virus infection, 175 patients with hepatitis $C$ virus infection, and 96 patients with other forms of liver disease. In 57 patients, HCC had been newly diagnosed, whereas in 257 patients it had recurred. There were 295 patients with a history of treatment for HCC and 19 patients with no such history. The mean initial sorafenib dose was $391.7 \pm 159 \mathrm{mg} /$ day (range: 200-800).

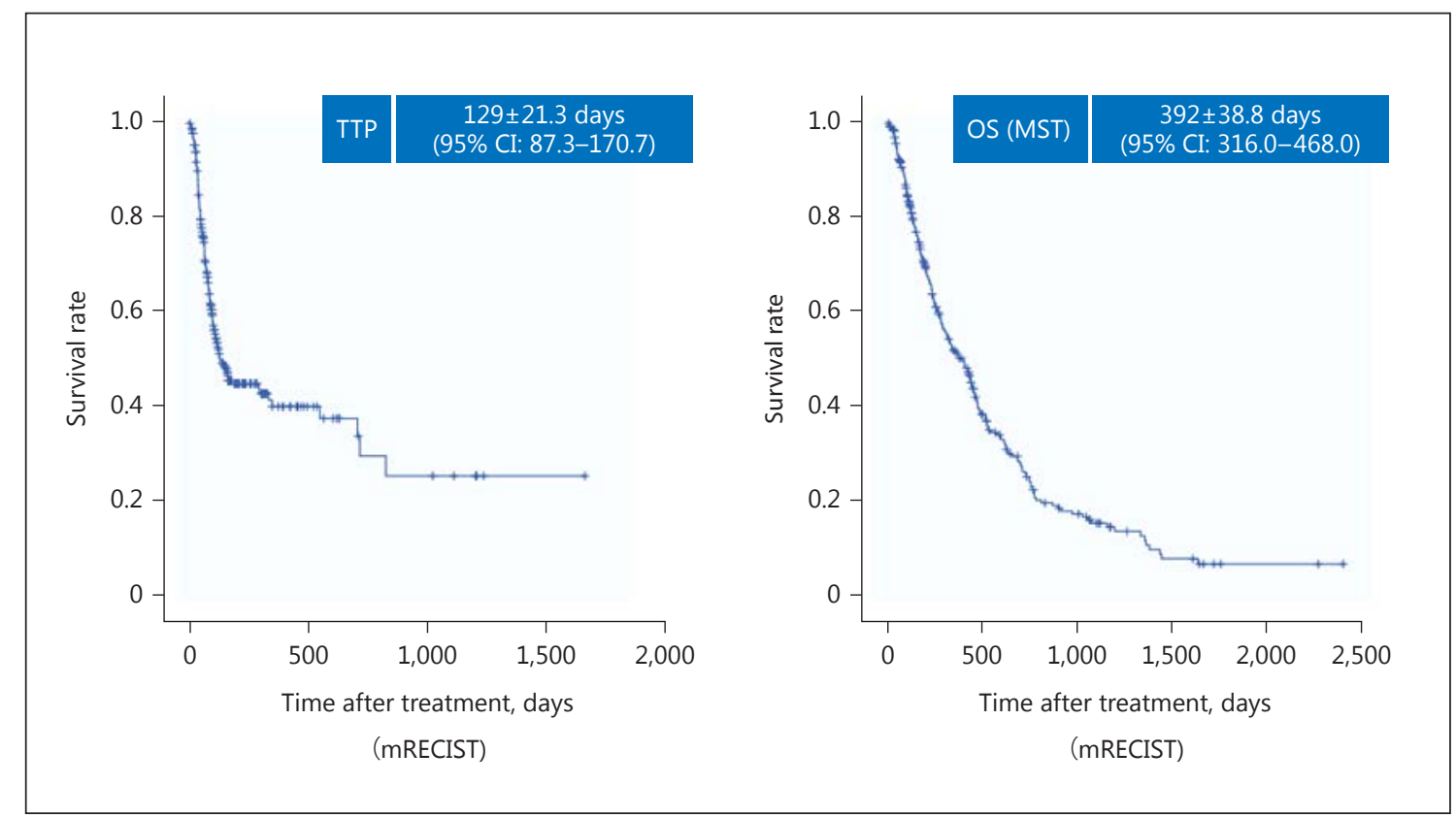

Fig. 1. Kaplan-Meier curves of time to progression (TTP) and overall survival (OS) of patients with sorafenib treatment. MST, median survival time; mRECIST, modified Response Evaluation Criteria in Solid Tumors. 
Table 1. Patient characteristics by Child-Pugh class

\begin{tabular}{llll}
\hline & $\begin{array}{l}\text { Child-Pugh class A } \\
(n=181)\end{array}$ & $\begin{array}{l}\text { Child-Pugh class B } \\
(n=55)\end{array}$ & $p$ \\
\hline Sex (male/female) & $196 / 44$ & $58 / 16$ & $\mathrm{~ns}$ \\
Age $(<70 / \geq 70$ years $)$ & $82 / 158$ & $37 / 37$ & 0.014 \\
Height $(<160 / \geq 160 \mathrm{~cm})$ & $94 / 140$ & $29 / 45$ & $\mathrm{~ns}$ \\
Weight $(<60 / \geq 60 \mathrm{~kg})$ & $140 / 94$ & $43 / 31$ & $\mathrm{~ns}$ \\
Background $(\mathrm{HBV} / \mathrm{HCV} /$ other $)$ & $33 / 130 / 77$ & $10 / 45 / 19$ & $\mathrm{~ns}$ \\
Initial manifestation/recurrence & $39 / 201$ & $18 / 56$ & $\mathrm{~ns}$ \\
Tumors $(<4 / \geq 5)$ & $114 / 115$ & $29 / 45$ & $\mathrm{~ns}$ \\
AFP $(<200 / \geq 200 \mathrm{ng} / \mathrm{mL})$ & $128 / 108$ & $34 / 37$ & $\mathrm{~ns}$ \\
DCP $(<400 / \geq 400 \mathrm{mAU} / \mathrm{mL})$ & $110 / 125$ & $24 / 44$ & $\mathrm{~ns}$ \\
AFP-L3 $(<10 / \geq 10 \%)$ & $39 / 87$ & $8 / 25$ & $\mathrm{~ns}$ \\
Stage $(\mathrm{III}$ or lower/IVa/IVb) & $106 / 40 / 94$ & $36 / 18 / 20$ & \\
\hline
\end{tabular}

AFP, $\alpha$-fetoprotein; DCP, des-gamma-carboxyprothrombin.

The Child-Pugh class and score at the initiation of sorafenib therapy was A-5 points in 129 patients, A-6 points in 111 patients, B-7 points in 48 patients, and B-8 points or higher in 26 patients. Sorafenib therapy was initiated in stage I in 6 patients, stage II in 51 patients, stage III in 85 patients, stage IVa in 58 patients, and stage IVb in 114 patients. The mean observation period in the present study was $223 \pm 277$ days (range: $2-1,665$ ). The indications for starting sorafenib were distal metastasis in $39.8 \%$ of the patients, unresponsiveness to TACE in $39.5 \%$, inability to perform TACE in $8.9 \%$, severe vascular invasion in $10.8 \%$, and other reasons in $1.0 \%$. The lung was the site of distant metastasis in 54 patients, the bone in 39 patients, lymph nodes in 36 patients, the adrenal glands in 14 patients, and the peritoneal cavity in 13 patients (some patients had metastases to multiple sites).

\section{OS and Radiological Evaluation}

The results of sorafenib therapy were a time to progression of $129 \pm 21.3$ days (95\% CI: 87.3-170.7) and a median OS (median survival time [MST]) of $392 \pm 38.8$ days (95\% CI: 316.0-468.0) (Fig. 1). A complete response, partial response, stable disease, progressive disease, and incomplete evaluation were observed in $10,25,113,147$, and 19 patients, respectively. The overall response rate was $11.1 \%$, and the tumor control rate was $47.4 \%$. An initial sorafenib dose of $200 \mathrm{mg}$ was administered to $77 \mathrm{pa}$ tients, $400 \mathrm{mg}$ to 202 patients (the most common dose), $600 \mathrm{mg}$ to 6 patients, and $800 \mathrm{mg}$ to 29 patients. The mean duration of sorafenib administration was 223.6 days, and the mean durations for the 200-, 400-, 600-, and 800-mg doses were $212.6,242.2,155$, and 136.8 days, respectively.

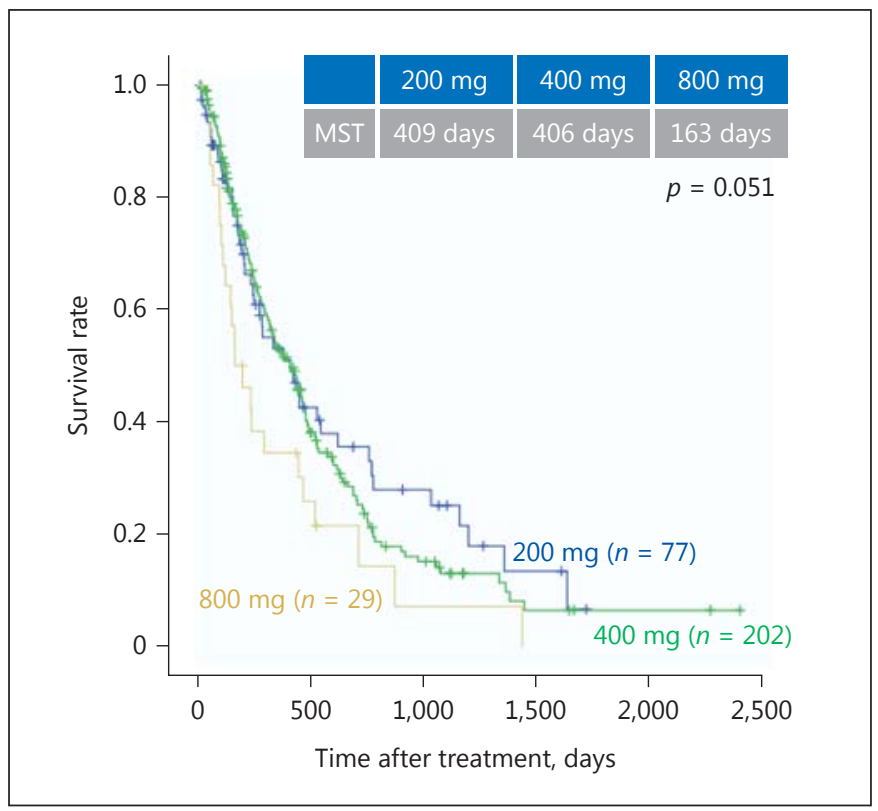

Fig. 2. Kaplan-Meier curves of overall survival (median survival time [MST]) of the patients in the standard-dose group, the halfdose group, and the quarter-dose group (by initial dose).

Disparities between institutions regarding the initial sorafenib dose were observed. The survival rate with a 400-mg initial dose was significantly higher than that with an 800-mg initial dose (Fig. 2).

A significant difference in patient characteristics according to Child-Pugh class was only observed for age ( $<70$ years, $\geq 70$ years; $p=0.014$ ); no significant differences were observed for the other variables (Table 1). Re- 


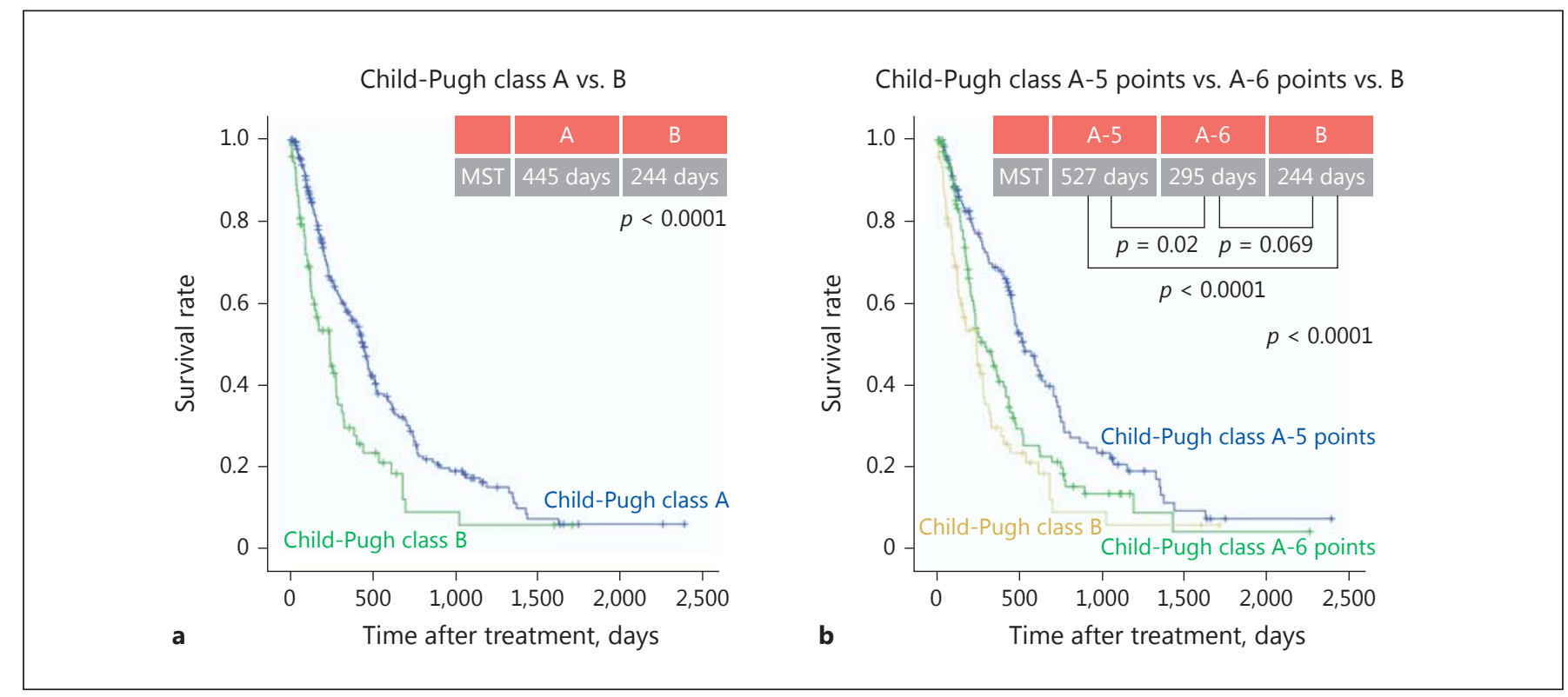

Fig. 3. Survival curves by Child-Pugh class and score at the start of therapy. a Kaplan-Meier curves of overall survival (median survival time [MST]) of the patients with Child-Pugh class A and Child-Pugh class B hepatocellular carcinoma (HCC). There was a significant difference between class A and class B. b Kaplan-Meier curves of overall survival (MST) of the patients with Child-Pugh

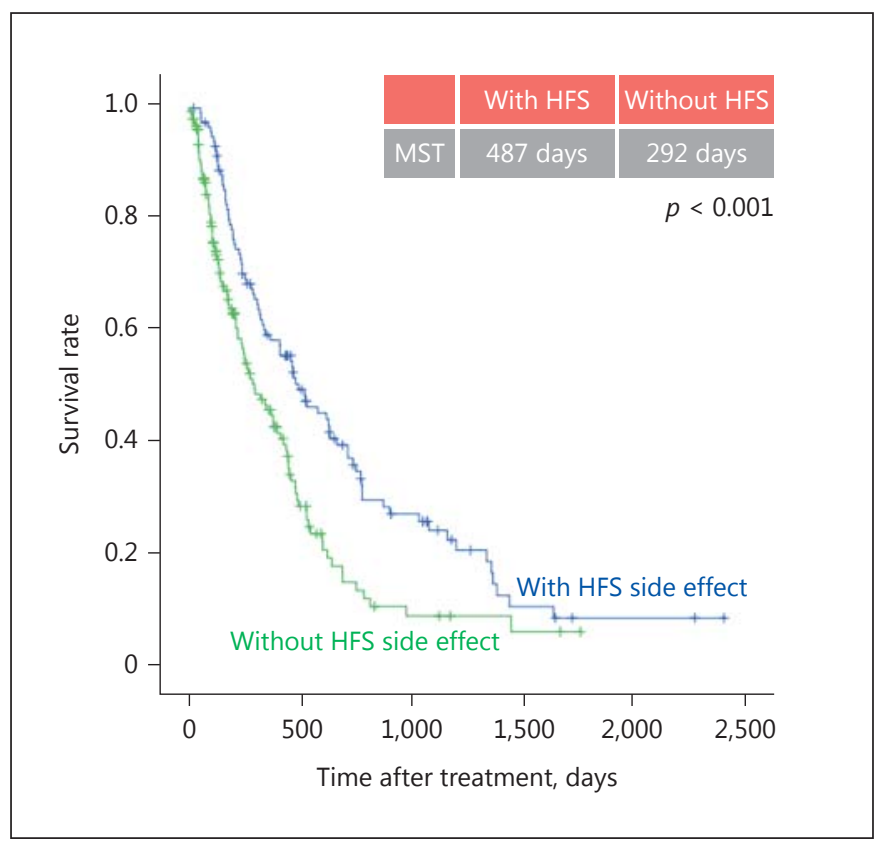

Fig. 4. Kaplan-Meier curves of overall survival (median survival time $[\mathrm{MST}]$ ) of the patients with a hand-foot syndrome (HFS) side effect and those without an HFS side effect. Overall survival was significantly longer among the patients who exhibited HFS as a side effect. class A-5 points and Child-Pugh class A-6 points. There was a significant difference between class A-5 points and class A- 6 points. Child-Pugh class B HCC had worse results than class A HCC, but the results for class A-6 points were close to those for class B (no significant difference between class A-6 points and class B).

garding survival rate, a significant difference was observed between Child-Pugh class A and class B, with the former being better $(p<0.0001)$. Further, OS was significantly different between Child-Pugh class A-5 points and class A- 6 points, with class A- 5 points being better ( $p=$ $0.02)$; however, OS was not significantly different between Child-Pugh class A- 6 points and class B $(p=0.069)$. The best OS was observed when treatment was initiated at Child-Pugh class A-5 points (Fig. 3).

Next, we examined the onset of hand-foot syndrome (HFS), which is an adverse effect of sorafenib. During the observation period, HFS was observed in 118 patients (37.6\%), with the mean number of days until onset being $29.6 \pm 33.8$ (range: 1-227). By grade, adverse effects were observed in 43 patients with grade I HCC (14.0\%), 42 patients with grade II HCC (13.8\%), and 24 patients with grade III HCC (7.9\%). The number of days until onset of HFS was less than 1 month from initiation of sorafenib therapy for $65.3 \%$ of the patients who exhibited HFS. Regarding differences in OS based on the presence or absence of HFS, the MST of the patients with HFS was 487 days, while that of the patients without HFS was 292 days; this difference was statistically significant $(p=0.001)$ (Fig. 4). The MSTs of the patients who underwent post-
116

Oncology 2017;93(suppl 1):113-119 DOI: $10.1159 / 000481241$
Ogawa et al. 


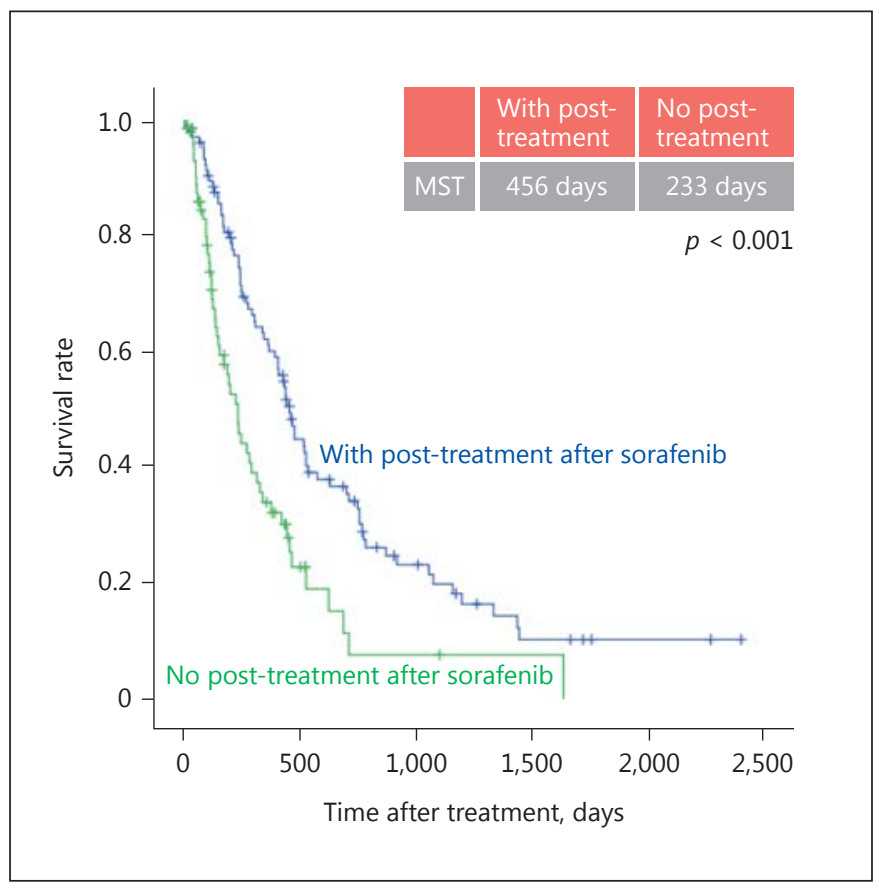

Fig. 5. Kaplan-Meier curves of overall survival (median survival time $[\mathrm{MST}]$ ) of the patients who received post-treatment after sorafenib therapy and those who did not. Overall survival was significantly longer among the patients who received post-treatment after sorafenib therapy.

progression treatment after sorafenib therapy and of those who did not were 456 and 233 days, respectively, with those who underwent post-progression treatment exhibiting a better OS $(p<0.001)$ (Fig. 5).

\section{Discussion}

Sorafenib is a multitargeted tyrosine kinase inhibitor that suppresses tumor proliferation and angiogenesis. This agent carries a mild-to-moderate toxicity profile and exhibits antitumor activity against various solid tumors [17]. It was the first systemic agent that was proven to prolong the survival of patients with advanced HCC in two phase III trials $[2,18]$, and it is now the standard of care for systemically treated patients $[5,19-22]$. The initial sorafenib dose recommended in the Sorafenib Hepatocellular Carcinoma Assessment Randomized Protocol (SHARP) trial and the Asia-Pacific trial was $800 \mathrm{mg} /$ day, but since sorafenib came into clinical use, there have been reports of lower initial doses. A large trial conducted among Japanese people, who have a smaller body surface area than Europeans and Americans, reported little dif-

Hand-Foot Syndrome and

Post-Treatment after Sorafenib ference between starting at 400 and $800 \mathrm{mg} /$ day. In the roughly 7 years since sorafenib was approved in Japan, several studies have reported using other initial doses besides $800 \mathrm{mg}$.

Some have also reported administering sorafenib to patients other than those with Child-Pugh class A disease $[23,24]$. In addition, better therapeutic results have been reported in patients who exhibited HFS, compared to those who did not $[25,26]$. In the present study, in which we examined the outcomes of sorafenib administration in 314 patients at 5 general hospitals in Kagawa Prefecture, Japan, the results of the present study also showed comparable results.

In the present study, only about $10 \%$ of the patients started at an initial dose of $800 \mathrm{mg}$, but their median OS was 392 days, which is better than the results of the SHARP and Asia-Pacific trials. Regarding liver function at the start of treatment, about one-fourth of the patients had Child-Pugh class B disease. The good OS results achieved even against a background of poor liver function are thought to have been largely because administration had started at an optimal stage for some patients and because of the benefits of post-progression treatment after sorafenib therapy, as the importance of post-progression treatment after sorafenib has already been reported [27].

Moreover, methods of preventing and treating HFS have been established since sorafenib came into clinical use, so the results were likely less affected by dropouts due to HFS compared to when the drug was first approved. Previously, patients who exhibited HFS had better results than those who did not; a similar trend was observed in the present study.

Regarding liver function at the start of therapy, in the GIDEON (Global Investigation of Therapeutic Decisions in Hepatocellular Carcinoma and of Its Treatment with Sorafenib) trial the group of Child-Pugh class A patients had better OS than the group that started at ChildPugh class B [28]; this was also observed in the present study. In the present study, even among the Child-Pugh class A patients, the group that started at class A-5 points had a significantly better OS than the group that started at class A-6 points; however, the OS of the group that started at class A-6 points was not significantly different from that of the Child-Pugh class B group. This suggests that among Child-Pugh class A patients, starting sorafenib when liver functions are well preserved, such as at class A-5 points, is preferable. The positive results observed in the present study for patients who underwent post-progression treatment such as TACE, hepatic arte-

Oncology 2017;93(suppl 1):113-119 117 
rial infusion chemotherapy, or ablation [29-32] after sorafenib therapy also indicate that initiating therapy when liver function is well preserved (Child-Pugh class A-5 points) is beneficial.

\section{Conclusion}

The best results were observed when therapy was initiated at Child-Pugh class A-5 points, which indicates that it is preferable to start sorafenib at the time when liver function is still well preserved. Starting sorafenib at a reduced dose in Japanese patients is also thought to lead to a better OS. Among the patients who exhibited HFS, those who were able to receive post-progression treatment after sorafenib therapy had good OS, which suggests that addressing this side effect is important for preserving the remaining hepatic reserve. The recent success of new systemic therapy agents $[33,34]$ and immunotherapies [35] will be expected to further improve survival among patients with advanced HCC.

\section{Disclosure Statement}

The authors have no conflicts of interest to declare.

\section{References}

1 McGlynn KA, Petrick JL, London WT: Global epidemiology of hepatocellular carcinoma: an emphasis on demographic and regional variability. Clin Liver Dis 2015;19:223-238.

2 Llovet JM, Ricci S, Mazzaferro V, Hilgard P, Gane E, Blanc JF, et al: Sorafenib in advanced hepatocellular carcinoma. N Engl J Med 2008; 359:378-390.

3 Kudo M, Izumi N, Kokudo N, Matsui O, Sakamoto M, Nakashima O, et al: Management of hepatocellular carcinoma in Japan: Consensus-Based Clinical Practice Guidelines proposed by the Japan Society of Hepatology (JSH) 2010 updated version. Dig Dis 2011;29:339-364.

4 Kudo M, Ueshima K: Positioning of a molecular-targeted agent, sorafenib, in the treatment algorithm for hepatocellular carcinoma and implication of many complete remission cases in Japan. Oncology 2010;78(suppl 1): 154-166.

5 Kudo M: Treatment of advanced hepatocellular carcinoma with emphasis on hepatic arterial infusion chemotherapy and molecular targeted therapy. Liver Cancer 2012;1:62-70.

6 Kudo M: Clinical practice guidelines for hepatocellular carcinoma differ between Japan, United States, and Europe. Liver Cancer 2015; 4:85-95.

7 Kudo M: Locoregional therapy for hepatocellular carcinoma. Liver Cancer 2015;4:163164.

8 Kudo M: Surveillance, diagnosis, treatment, and outcome of liver cancer in Japan. Liver Cancer 2015;4:39-50.

9 Tsurusaki M, Murakami T: Surgical and locoregional therapy of HCC: TACE. Liver Cancer 2015;4:165-175.
10 Arizumi T, Ueshima K, Minami T, Kono M, Chishina $\mathrm{H}$, Takita $\mathrm{M}$, et al: Effectiveness of sorafenib in patients with transcatheter arterial chemoembolization (TACE) refractory and intermediate-stage hepatocellular carcinoma. Liver Cancer 2015;4:253-262.

11 Geschwind JF, Gholam PM, Goldenberg A, Mantry P, Martin RC, Piperdi B, et al: Use of transarterial chemoembolization (TACE) and sorafenib in patients with unresectable hepatocellular carcinoma: US regional analysis of the GIDEON registry. Liver Cancer 2016;5:37-46.

12 Obi S, Sato S, Kawai T: Current status of hepatic arterial infusion chemotherapy. Liver Cancer 2015;4:188-199.

13 Lin CC, Hung CF, Chen WT, Lin SM: Hepatic arterial infusion chemotherapy for advanced hepatocellular carcinoma with portal vein thrombosis: impact of early response to 4 weeks of treatment. Liver Cancer 2015;4: 228-240.

14 Chen BB, Murakami T, Shih TT, Sakamoto M, Matsui O, Choi BI, et al: Novel imaging diagnosis for hepatocellular carcinoma: consensus from the 5th Asia-Pacific Primary Liver Cancer Expert Meeting (APPLE 2014). Liver Cancer 2015;4:215-227.

15 Joo I, Lee JM: Recent advances in the imaging diagnosis of hepatocellular carcinoma: value of gadoxetic acid-enhanced MRI. Liver Cancer 2016;5:67-87.

16 Lencioni R, Llovet JM: Modified RECIST (mRECIST) assessment for hepatocellular carcinoma. Semin Liver Dis 2010;30:52-60.

17 Strumberg D, Richly H, Hilger RA, Schleucher N, Korfee S, Tewes M, et al: Phase I clinical and pharmacokinetic study of the novel Raf kinase and vascular endothelial growth factor receptor inhibitor BAY 43-9006 in patients with advanced refractory solid tumors. J Clin Oncol 2005;23:965-972.
18 Cheng AL, Kang YK, Chen Z, Tsao CJ, Qin S, Kim JS, et al: Efficacy and safety of sorafenib in patients in the Asia-Pacific region with advanced hepatocellular carcinoma: a phase III randomised, double-blind, placebo-controlled trial. Lancet Oncol 2009;10:25-34.

19 Alves RC, Alves D, Guz B, Matos C, Viana M, Harriz M, et al: Advanced hepatocellular carcinoma. Review of targeted molecular drugs. Ann Hepatol 2011;10:21-27.

20 Peck-Radosavljevic M: Drug therapy for advanced-stage liver cancer. Liver Cancer 2014; 3:125-131.

21 Keating GM, Santoro A: Sorafenib: a review of its use in advanced hepatocellular carcinoma. Drugs 2009;69:223-240.

22 Saraswat VA, Pandey G, Shetty S: Treatment algorithms for managing hepatocellular carcinoma. J Clin Exp Hepatol 2014;4(suppl 3): S80-S89.

23 Nishikawa H, Osaki Y, Endo M, Takeda H, Tsuchiya K, Joko K, et al: Comparison of standard-dose and half-dose sorafenib therapy on clinical outcome in patients with unresectable hepatocellular carcinoma in field practice: a propensity score matching analysis. Int J Oncol 2014;45:2295-2302.

24 Morimoto M, Numata K, Kondo M, Kobayashi S, Ohkawa S, Hidaka H, et al: Field practice study of half-dose sorafenib treatment on safety and efficacy for hepatocellular carcinoma: a propensity score analysis. Hepatol Res 2015;45:279-287.

25 Vincenzi B, Santini D, Russo A, Addeo R, Giuliani F, Montella L, et al: Early skin toxicity as a predictive factor for tumor control in hepatocellular carcinoma patients treated with sorafenib. Oncologist 2010;15:85-92. 
26 Otsuka T, Eguchi Y, Kawazoe S, Yanagita K, Ario K, Kitahara K, et al: Skin toxicities and survival in advanced hepatocellular carcinoma patients treated with sorafenib. Hepatol Res 2012;42:879-886.

27 Arizumi T, Ueshima K, Takeda H, Osaki Y, Takita M, Inoue T, et al: Comparison of systems for assessment of post-therapeutic response to sorafenib for hepatocellular carcinoma. J Gastroenterol 2014;49:1578-1587.

28 Kudo M, Ikeda M, Takayama T, Numata K, Izumi N, Furuse J, et al: Safety and efficacy of sorafenib in Japanese patients with hepatocellular carcinoma in clinical practice: a subgroup analysis of GIDEON. J Gastroenterol 2016;51:1150-1160.
29 Kang TW, Rhim H: Recent advances in tumor ablation for hepatocellular carcinoma. Liver Cancer 2015;4:176-187.

30 Lencioni R, de Baere T, Martin RC, Nutting CW, Narayanan G: Image-guided ablation of malignant liver tumors: recommendations for clinical validation of novel thermal and non-thermal technologies - a Western perspective. Liver Cancer 2015;4:208-214.

31 Lin CC, Cheng YT, Chen MW, Lin SM: The effectiveness of multiple electrode radiofrequency ablation in patients with hepatocellular carcinoma with lesions more than $3 \mathrm{~cm}$ in size and Barcelona Clinic Liver Cancer stage A to B2. Liver Cancer 2016;5:8-20.
32 Kitai S, Kudo M, Nishida N, Izumi N, Sakamoto M, Matsuyama Y, et al: Survival benefit of locoregional treatment for hepatocellular carcinoma with advanced liver cirrhosis. Liver Cancer 2016;5:175-189.

33 Kudo M: Molecular targeted therapy for hepatocellular carcinoma: where are we now? Liver Cancer 2015;4:I-VII.

34 Kudo M: Regorafenib as second-line systemic therapy may change the treatment strategy and management paradigm for hepatocellular carcinoma. Liver Cancer 2016;5:235-244.

35 Kudo M: Immune checkpoint blockade in hepatocellular carcinoma. Liver Cancer 2015;4: 201-207. 